Celebrating 50 Years of Nonlinear Optics

\title{
Surface Nonlinear Optics
}

\author{
Y. Ron Shen \\ Physics Department \\ University of California \\ Berkeley, CA 94720
}




\section{Birth of Nonlinear Optics}

P.A. Franken, A.E. Hill, C.W. Peters, and G. Weinreich, "Generation of Optical Harmonics”, Phys. Rev. Lett. 7, 118 (August 15, 1961)

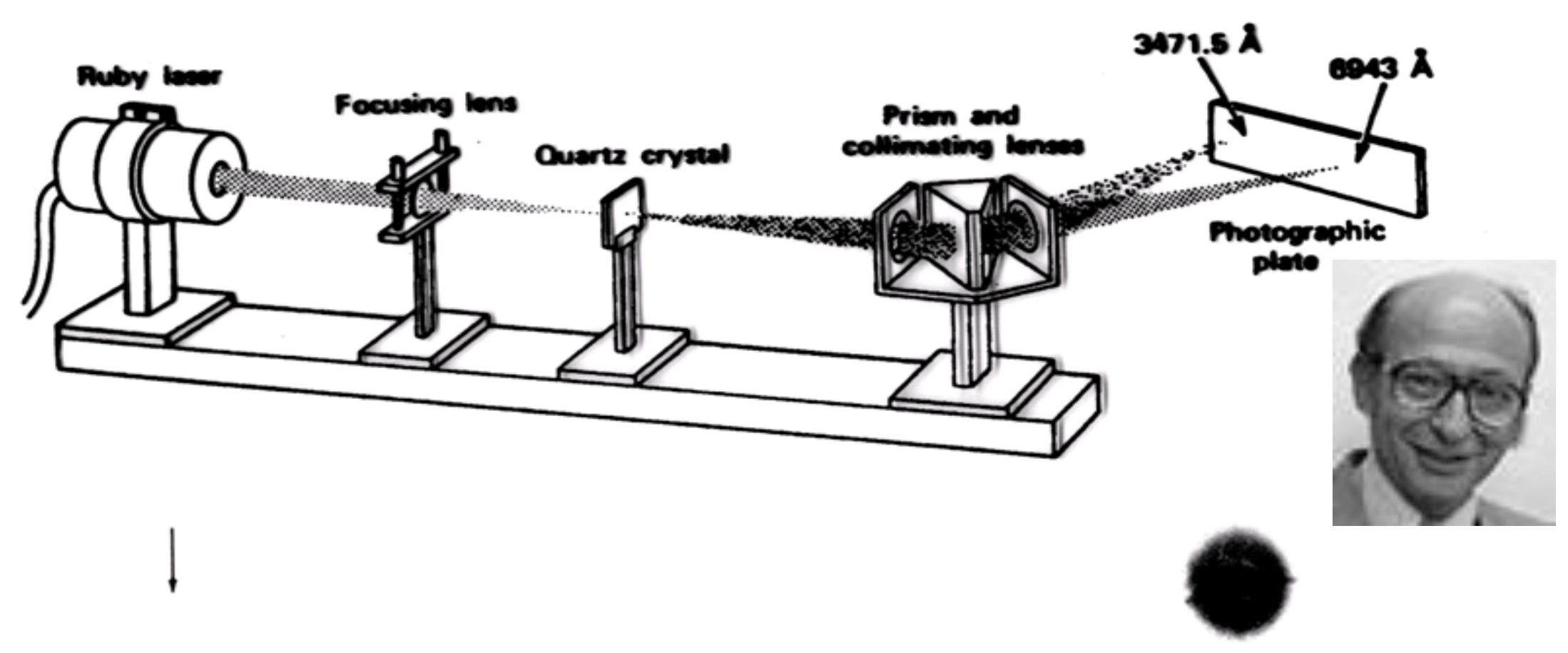




\section{Theoretical Foundation}

\section{Interactions between Light Waves in a Nonlinear Dielectric*}

J. A. Armstrong, N. Bloembergen, J. Ducuing, $†$ and P. S. Pershan

Division of Engineering and Applied Physics, Harvard University, Cambridge, Massachusetts

(Received April 16, 1962)

\section{Light Waves at the Boundary of Nonlinear Media}

N. Bloembergen and P. S. Pershan

Division of Engineering and Applied Physics, Harvard University, Cambridge, Massachusetts

(Received June 11, 1962)

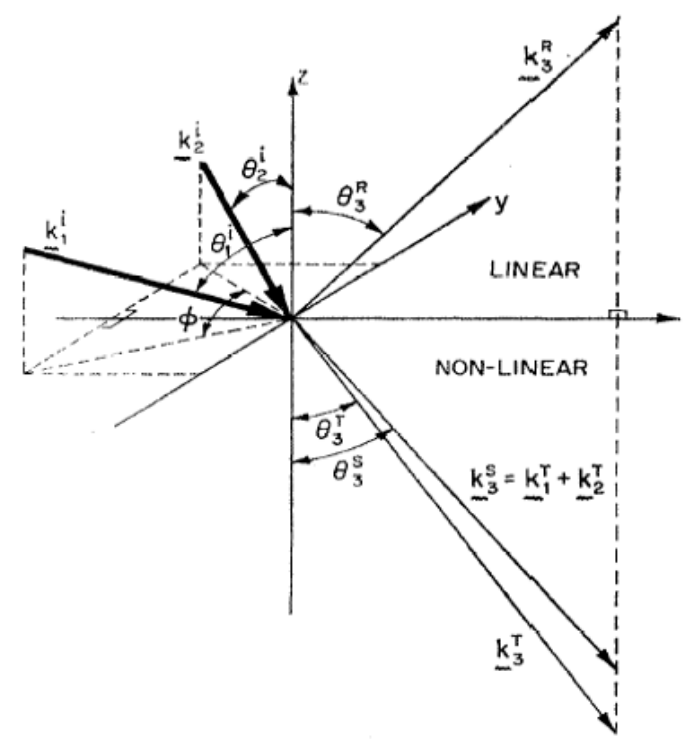




\section{OBSERVATION OF REFLECTED LIGHT HARMONICS AT THE BOUNDARY OF PIEZOELECTRIC CRYSTALS*}

J. Ducuing and N. Bloembergen

Division of Engineering and Applied Physics, Harvard University, Cambridge, Massachusetts (Received 2 May 1963)

Experimental Verification of the Laws for the Reflected Intensity of Second-Harmonic Light*

R. K. Chang and N. Bloevbergen

Gordon McKay Laboratory, Harvard University, Cambridge, Massachusetts

(Received 1 November 1965) 
Optical Second-Harmonic Generation in Reflection from Media with Inversion Symmetry*

N. Bloembergen, R. K. Chang, $\dagger$ S. S. Jha, and C. H. Lee: Division of Engineering and Applied Physics, Harvard University, Cambridge, Massachusetts (Received 6 May 1968)

Surface nonlinearity from field discontinuity at a surface

Optical Sceond Harmonic Generation from Submonolayer Na-Covered Ge Surfaces J.M. Chen, J,R. Bower, C.S. Wang, and C.H. Lee Optics Communications 9, 132, (1973)

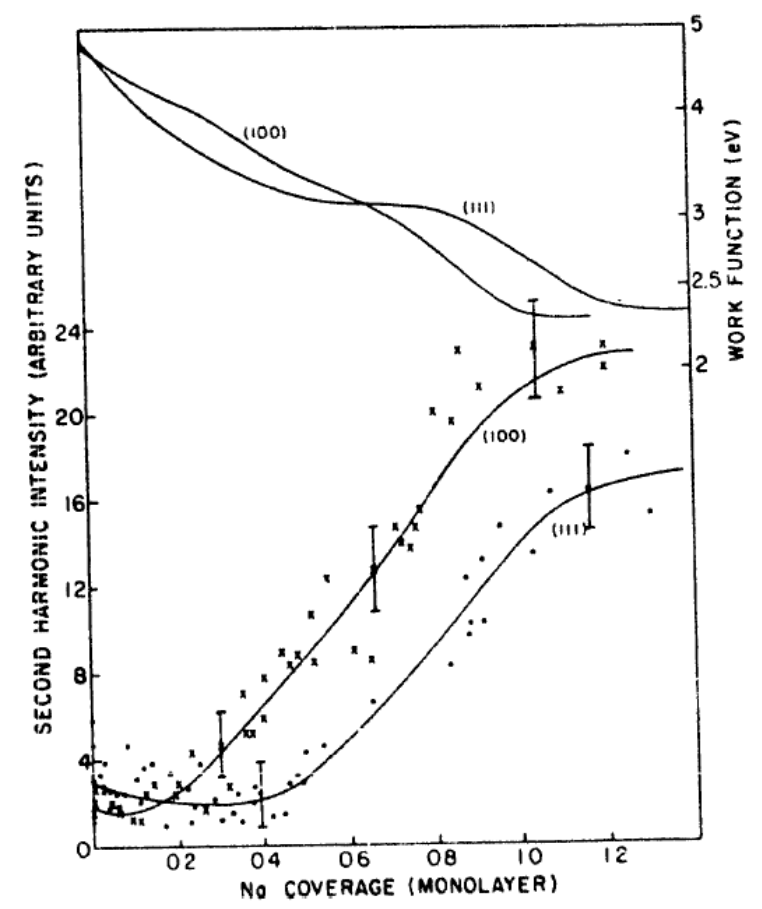




\section{Nonlinear Optics with Surface EM Waves: SHG by Colliding Surface Plasmons}
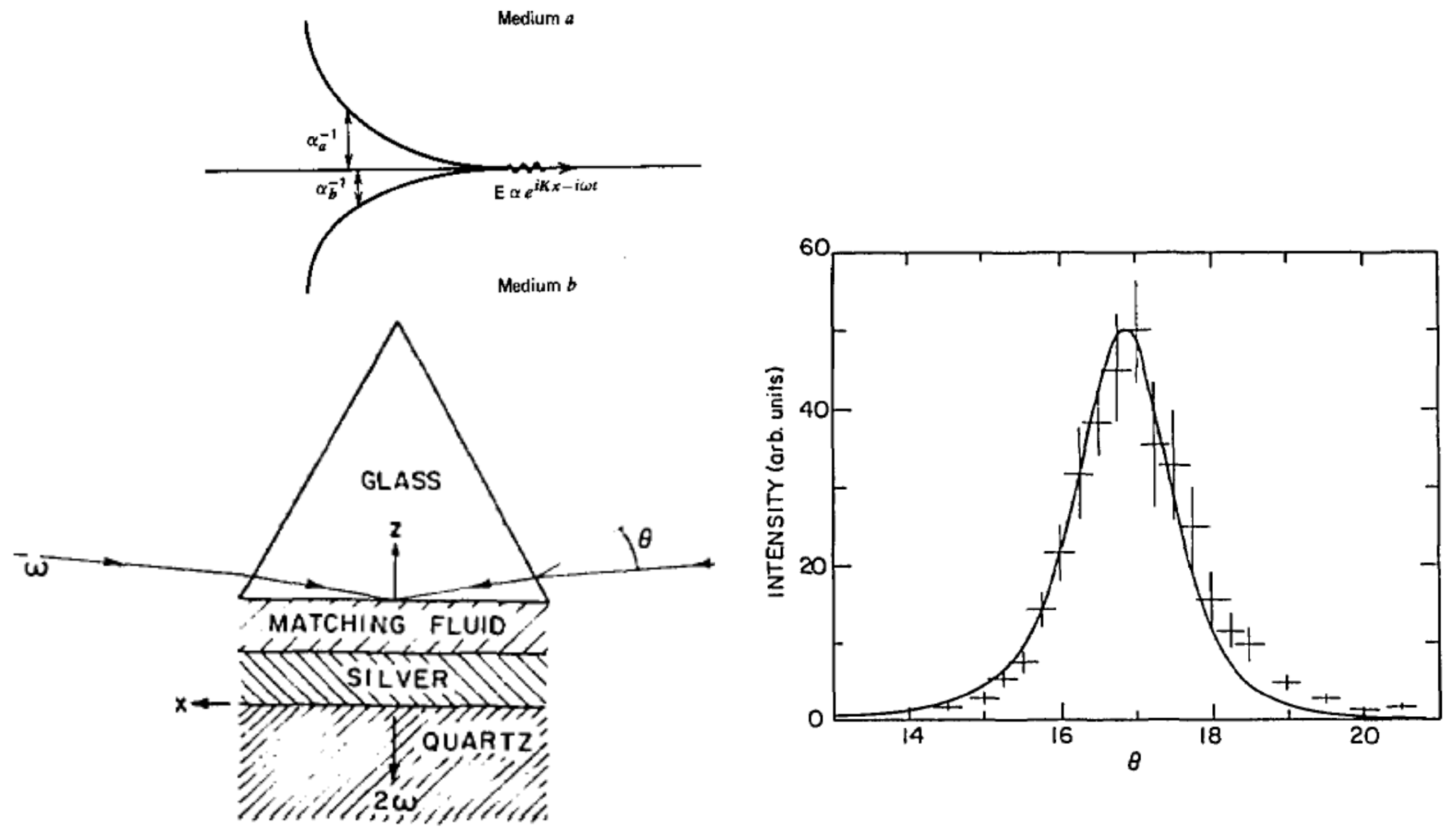

(c)

$$
k_{1 x}(\omega)+k_{2 x}(\omega)=k_{x}(2 \omega)=0
$$




\section{Nonlinear Optics \\ As Surface Probes}




\section{Surface-Enhanced Raman Scattering}

M. Fleischmann et al, Chem. Phys. Lett. 26, 163 (1974)

- Raman spectrum of adsorbed pyridine on roughed Ag electrode could be easily detected

- Enhancement of Raman signal was estimated to be $\sim 10^{6}$.

- Enhancement mechanism:

Raman probability $\propto \sigma_{R}\left|E_{l o c}\left(\omega_{l}\right)\right|^{2}\left|E_{l o c}\left(\omega_{s}\right)\right|^{2}$

$$
\propto \sigma_{R}\left|L\left(\omega_{l}\right)\right|^{2}\left|L\left(\omega_{s}\right)\right|^{2}\left|E\left(\omega_{l}\right)\right|^{2}\left|E\left(\omega_{s}\right)\right|^{2}
$$

Local field enhancement via surface plasmons in $L(\omega)$

Resonance enhancement due to charge-transfer excitation in $\sigma_{R}$ 


\section{Surface Enhancement}

\section{of Second Harmonic Generation}

C.K. Chen et al, PRL 46, 145 (1981)

$I(2 \omega) \propto\left|\chi^{(2)} E_{l o c}(\omega) E_{l o c}(\omega)\right|^{2} \propto|L(\omega)|^{4}\left|\chi^{(2)} E(\omega) E(\omega)\right|^{2}$

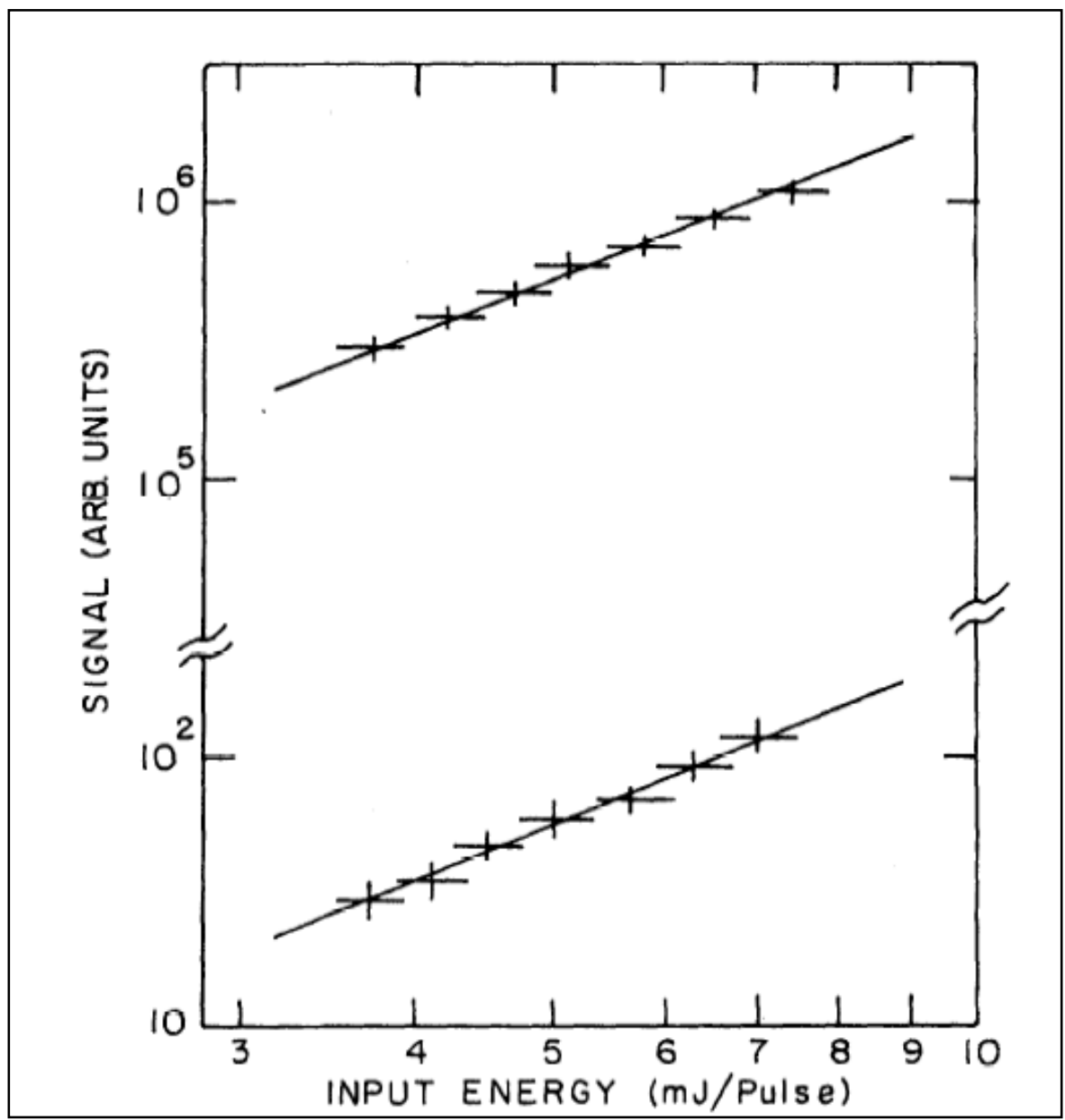




\section{SHG Detection of a Molecular Monolayer on $\mathrm{Ag}$ during an Electrolytic Cycle \\ Chen et al, PRL 46, 1010 (1981)}
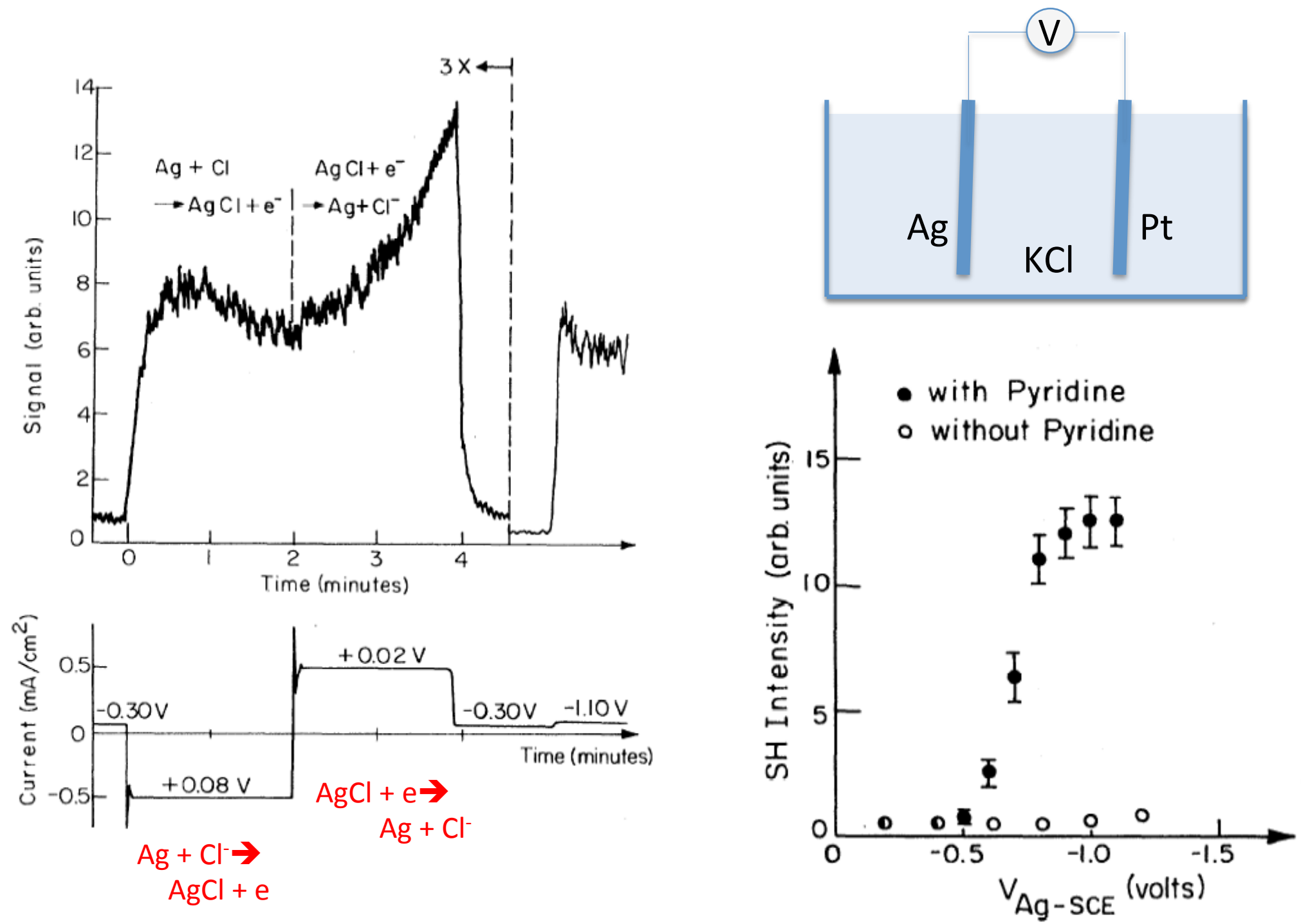


\section{Second-Harmonic Generation from Sub-Monolayer Molecular Adsorbates Using a CW Diode Laser: Maui Surface Experiment G.T.Boyd, Y.R.Shen, and T.W.Hansch}

This experiment was conceived at the Maui Surf Hotel, Kaanapali Beach, Maui, Hawaii. We wish to thank the hotel for its hospitality in accommodating the preparation of this experiment in the Queen Suite and the subsequent demonstration in its conference hall. 


\section{Spectrum of a Molecular Monolayer}

T.F.Heinz et al, PRL 48, 478 (1982)

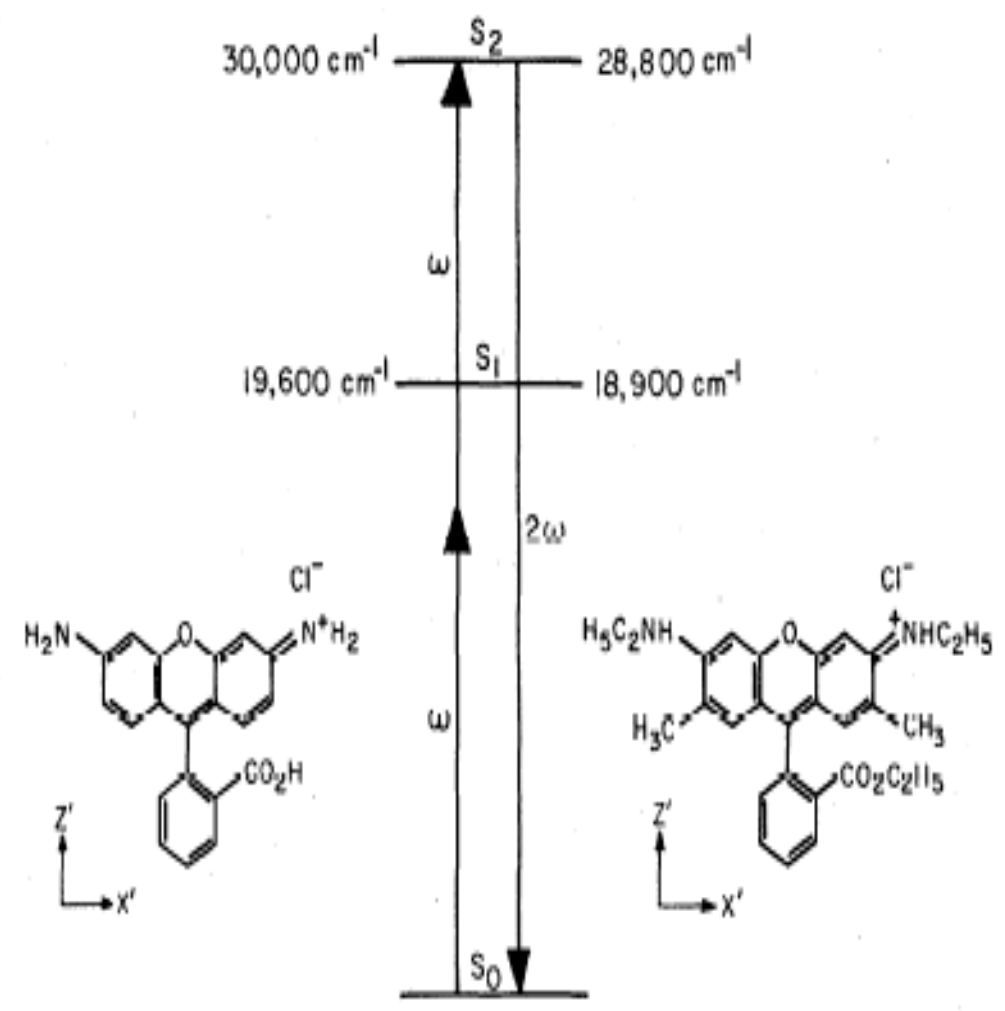

Rhodomine 110

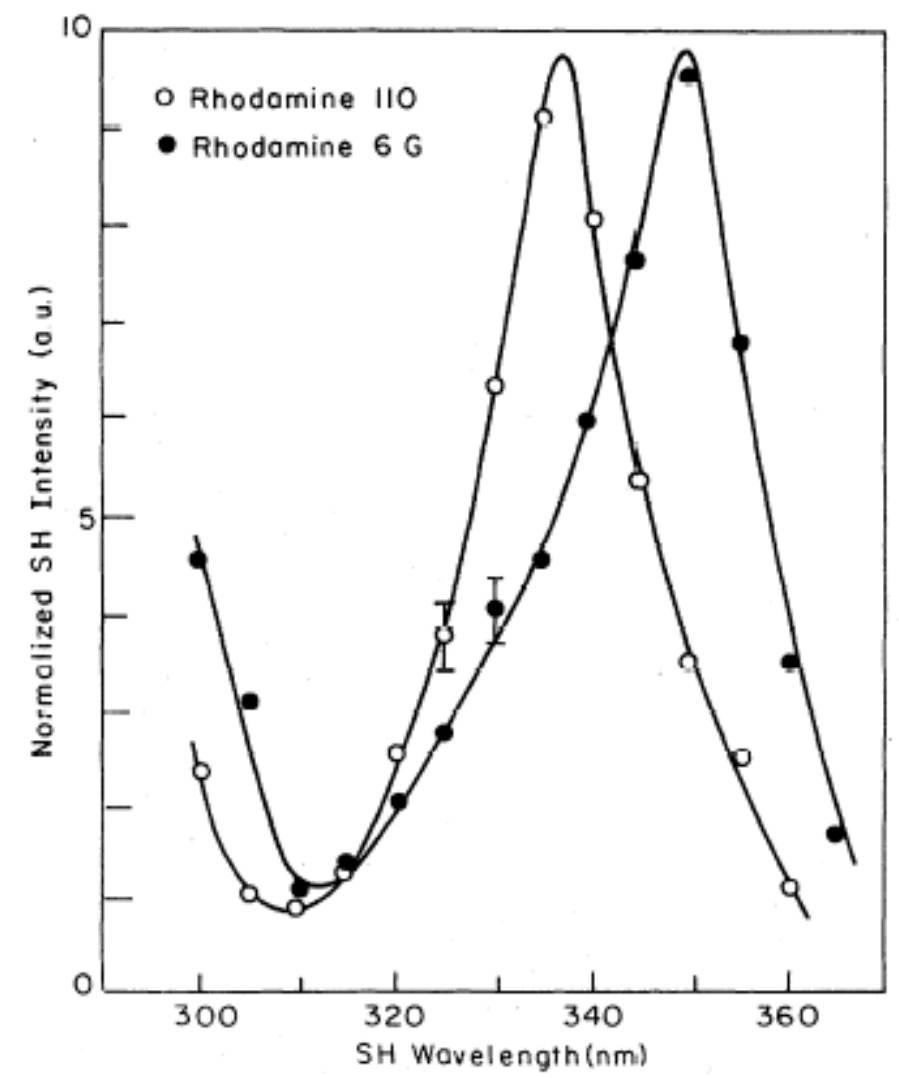




\section{Sum-Frequency Surface Spectroscopy}

$\vec{P}^{(2)}\left(\omega=\omega_{1}+\omega_{2}\right)=\vec{\chi}: \vec{E}\left(\omega_{1}\right) \vec{E}\left(\omega_{2}\right)$

$\vec{\chi}^{(2)}=0$ in media with inversion symmetry

$\neq 0$ at surfaces or interfaces
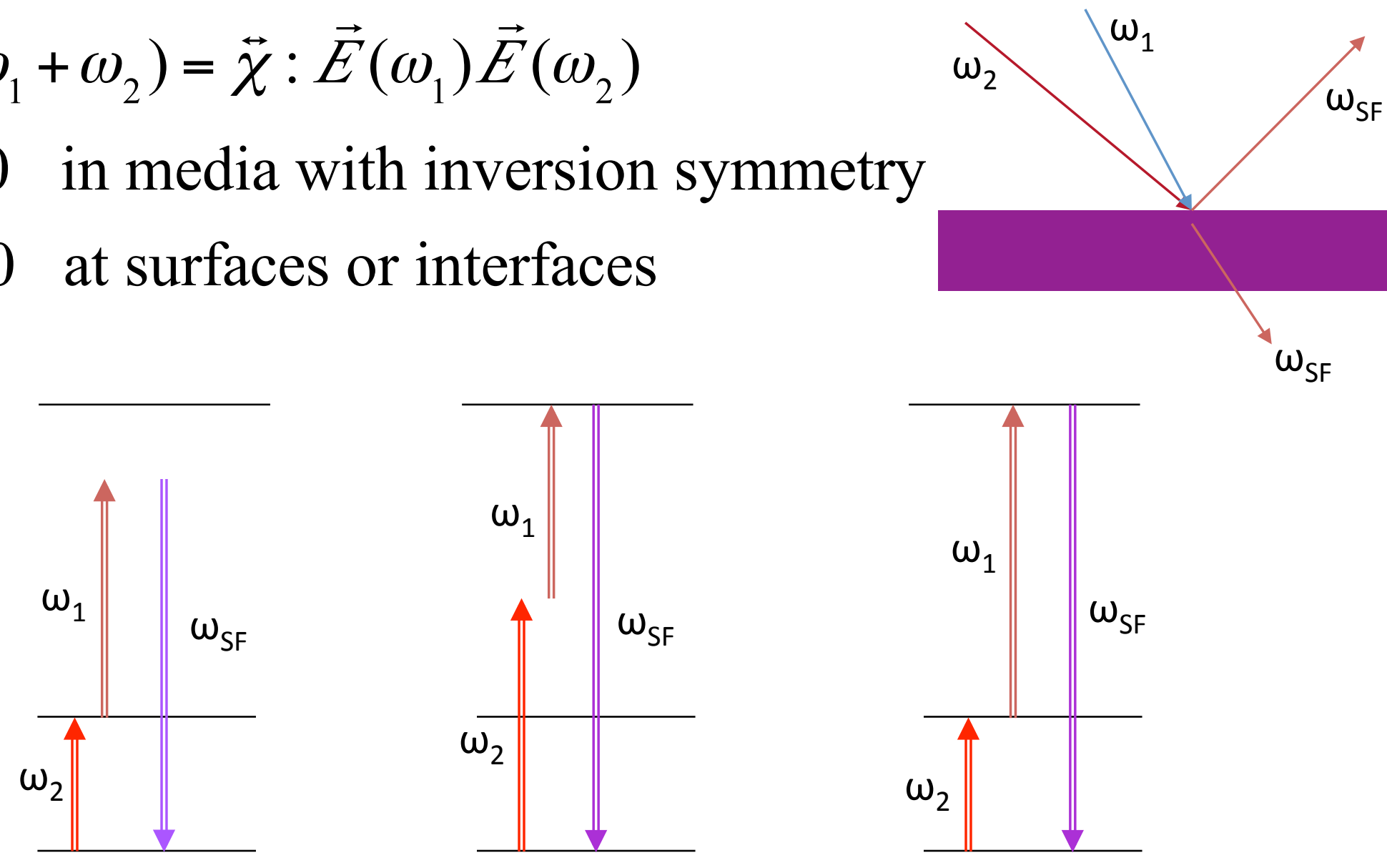

$$
\begin{aligned}
& \text { As } \omega_{2} \rightarrow \omega_{\mathrm{q}}, \text { or } \omega_{\mathrm{SF}} \rightarrow \omega_{\mathrm{el}}, \text { SFG is resonantly enhanced, } \\
& \rightarrow \rightarrow \text { Spectroscopic information. }
\end{aligned}
$$




\title{
Water Interfaces \\ Most important liquid in all aspects of life
}

\author{
Two Most Problems of the World
}

Water purification $\rightarrow$ Water resources

Water splitting $\rightarrow$ Energy resources

If we understand and know how to manipulate water interfaces, we can solve both problems.

But little is known about water interfaces 


\section{SF Spectrum (SSP) of OH Stretches at Vapor/Water Interface}
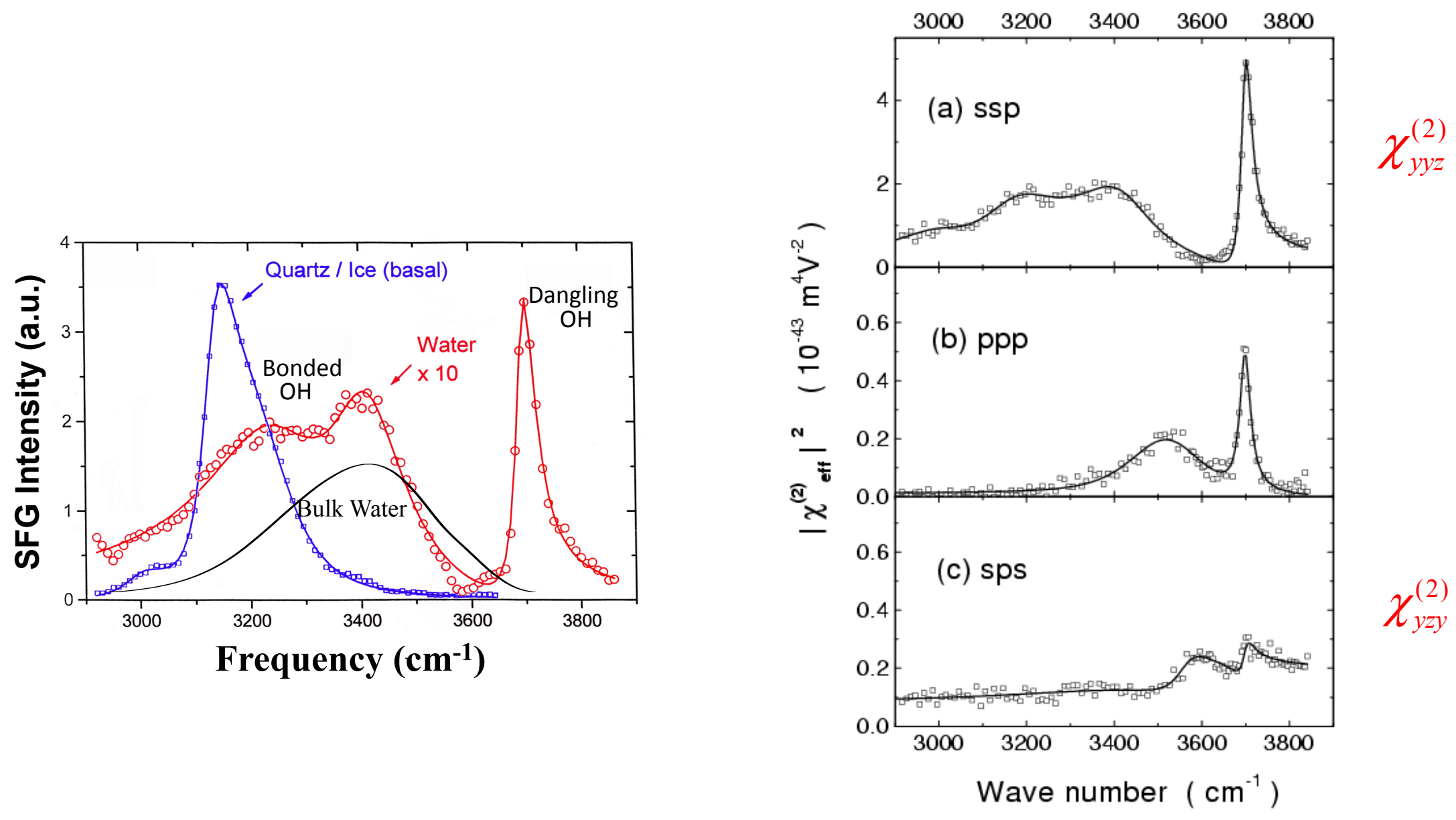
Free OH Peak, Ice-like Band, Strong Polarization Dependence $\rightarrow \rightarrow$ water interface has a highly distorted (dynamically varying) H-bonding structure, strongly disordered like bulk water, but partially ordered like ice.

Donor and acceptor bonds of water molecules
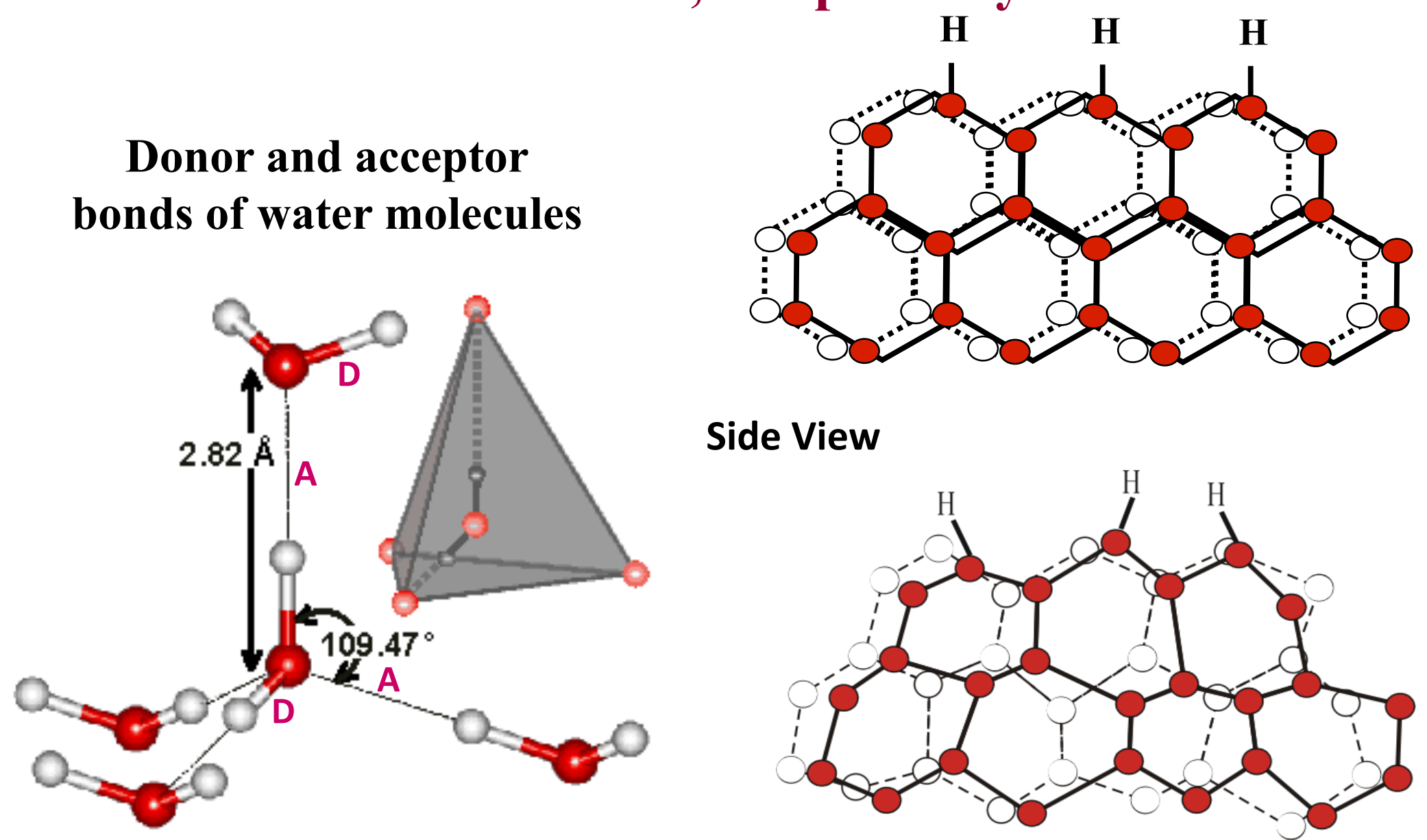

Side View

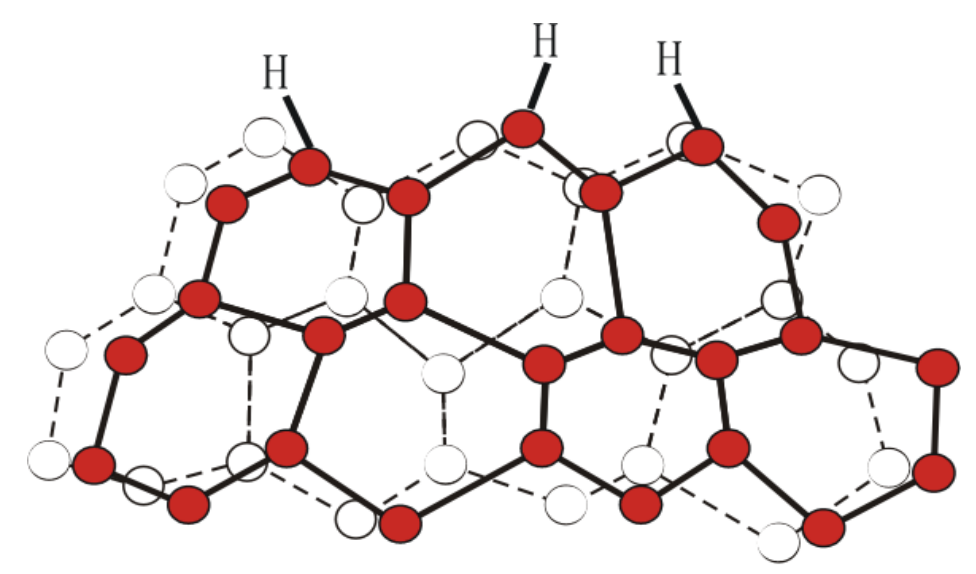




\section{More Structural Information from $\operatorname{Im} \chi_{S}^{(2)}$ Spectrum}

Resonances are better characterized by $\operatorname{Im} \chi_{S}^{(2)}$, analogous to absorption and emission spectra.

$$
\operatorname{Im} \chi_{S}^{(2)}>0
$$

net polar orientation

$$
\begin{aligned}
& \text { of O-H up } \\
& \operatorname{Im} \chi_{S}^{(2)}<0
\end{aligned}
$$

net polar orientation of O-H down
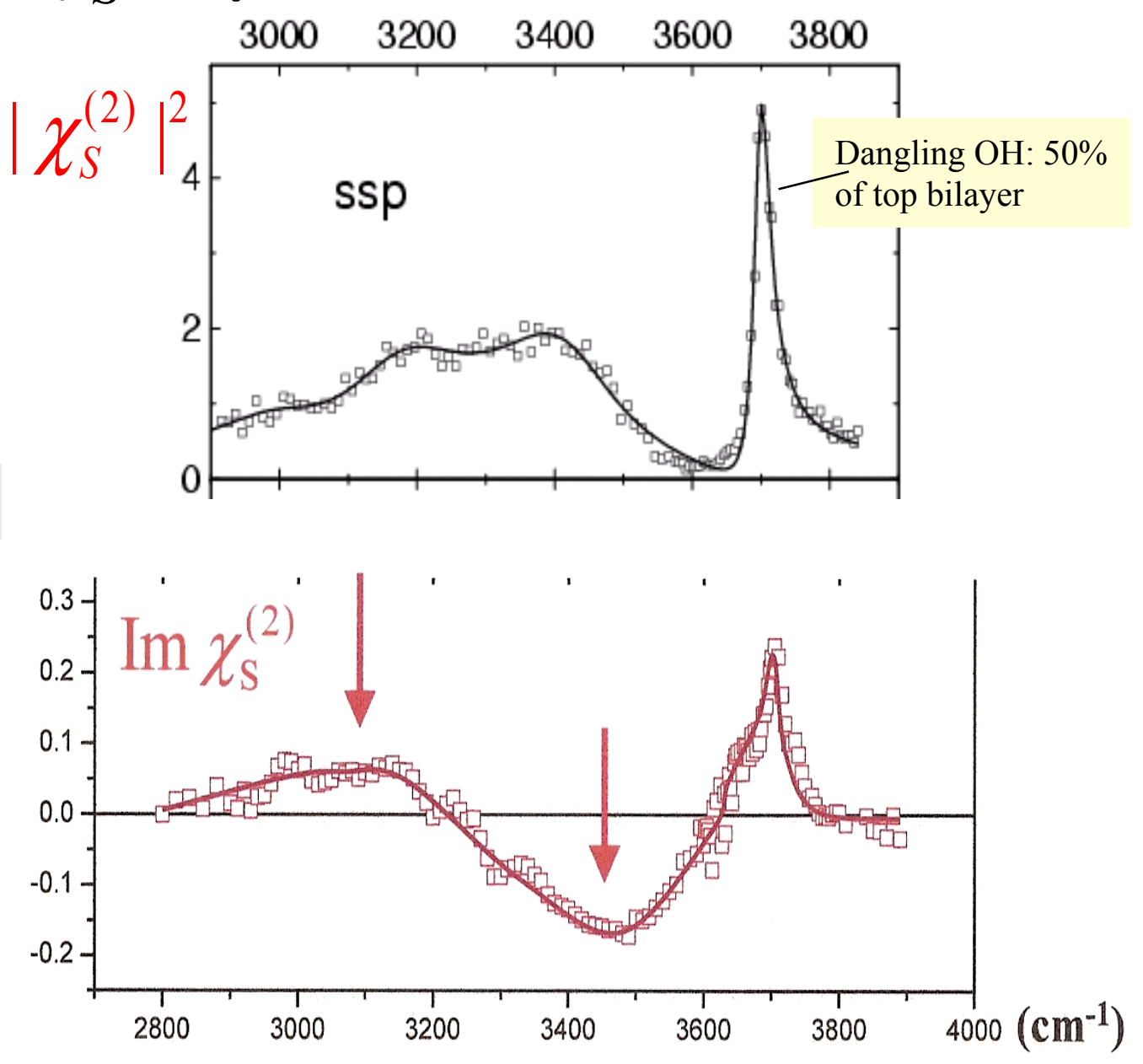

Frequency $\left(\mathrm{cm}^{-1}\right)$ 


\section{Physical Understanding of the $\operatorname{Im} \chi_{S}^{(2)}$ Spectrum}
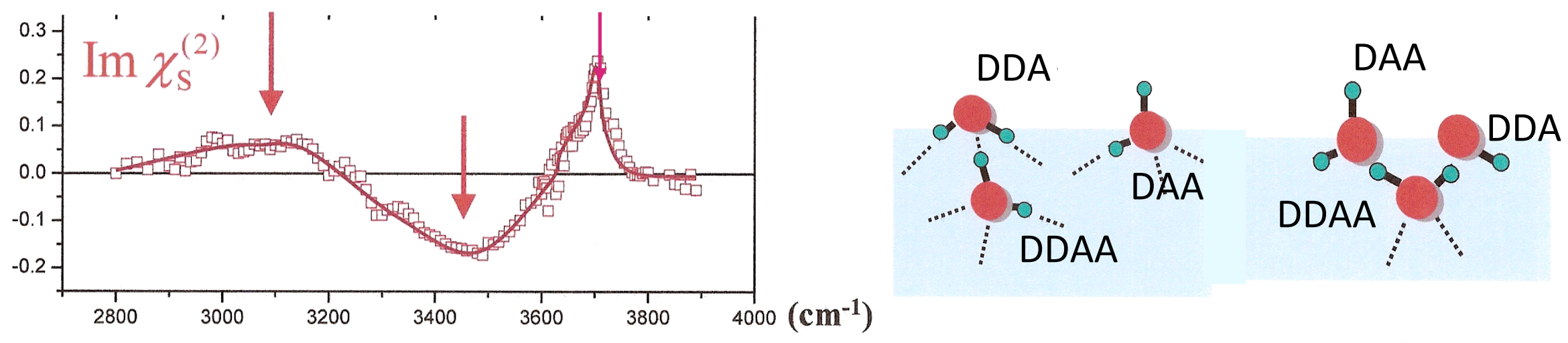

- Interface is composed of DDA, DAA, and DDAA molecules with varying $\mathrm{H}$-bonding strength.

- Free $\mathrm{OH}$ of DAA contributes to the peak at $3720 \mathrm{~cm}^{-1}$.

- Downward pointing OHs of DDA and DAA in the top layer contribute to the negative band.

- DDAA straddling DDA and DAA in the top layer with strong symmetric donor bonds contribute to the positive band.

- More loosely bonded DDAA also have net contribution to the negative band. 


\section{Ultrafast Surface Dynamics at Water Interfaces}

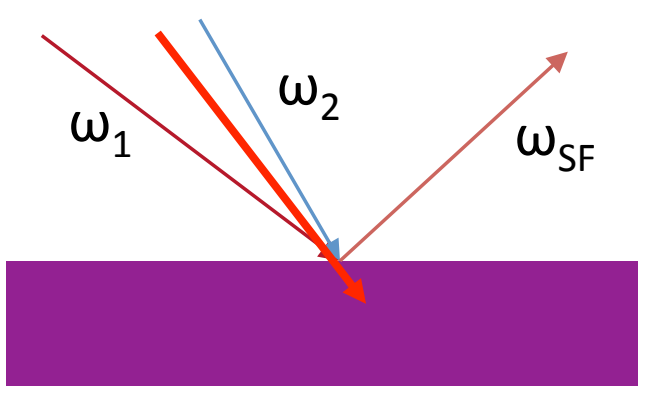

- Pump: fs pulse excitations of $\mathrm{OH}$ stretch vibrations (of both surface and bulk)

- Probe: time-resolved surface-specific SF vibrational spectroscopy to probe time evolution of the vibrational spectrum of water interface. 


\section{Spectral Hole-Burning at Water/Silica Interface}

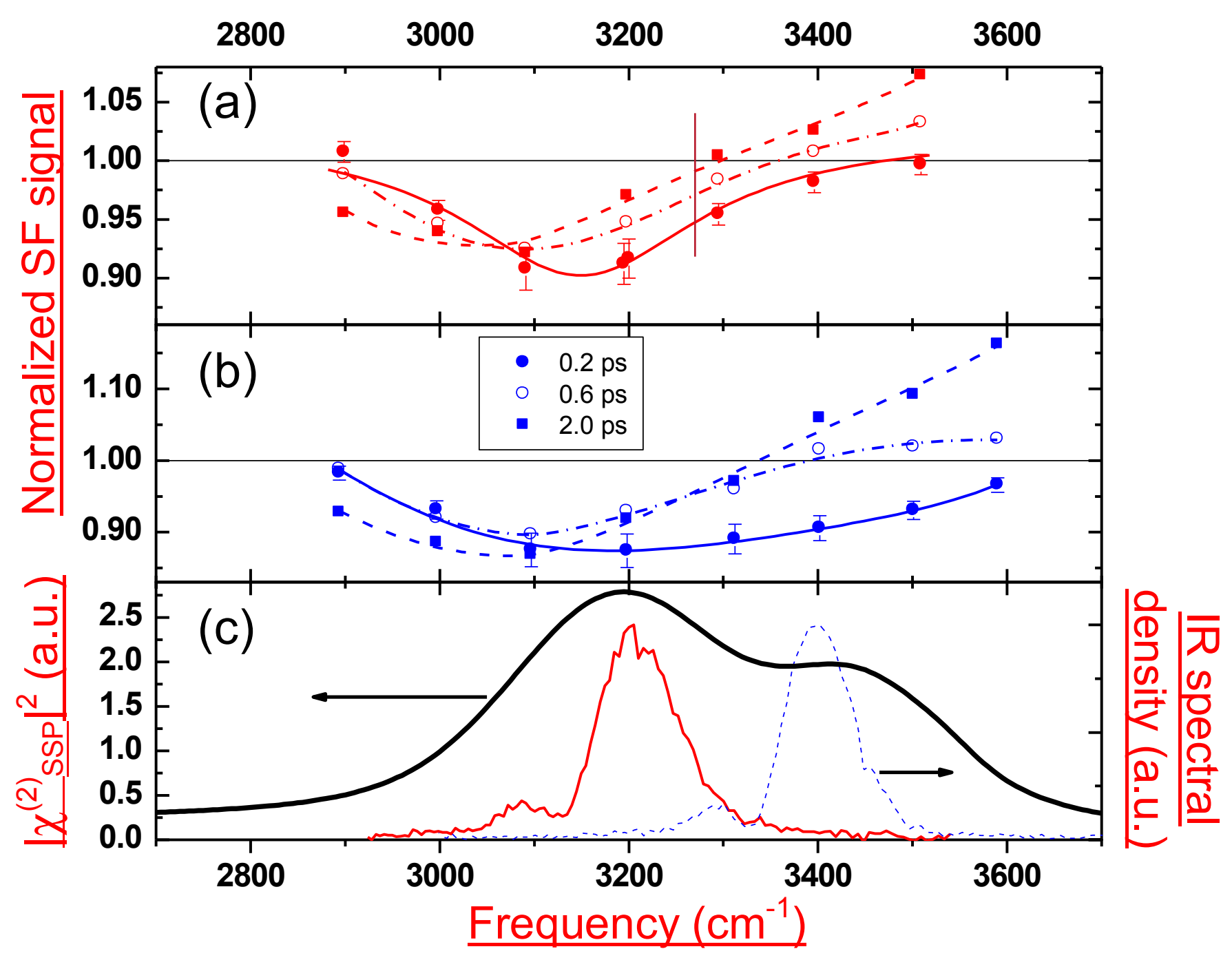




\section{Time-Resolved SF Probing of Spectral Hole Recovery}

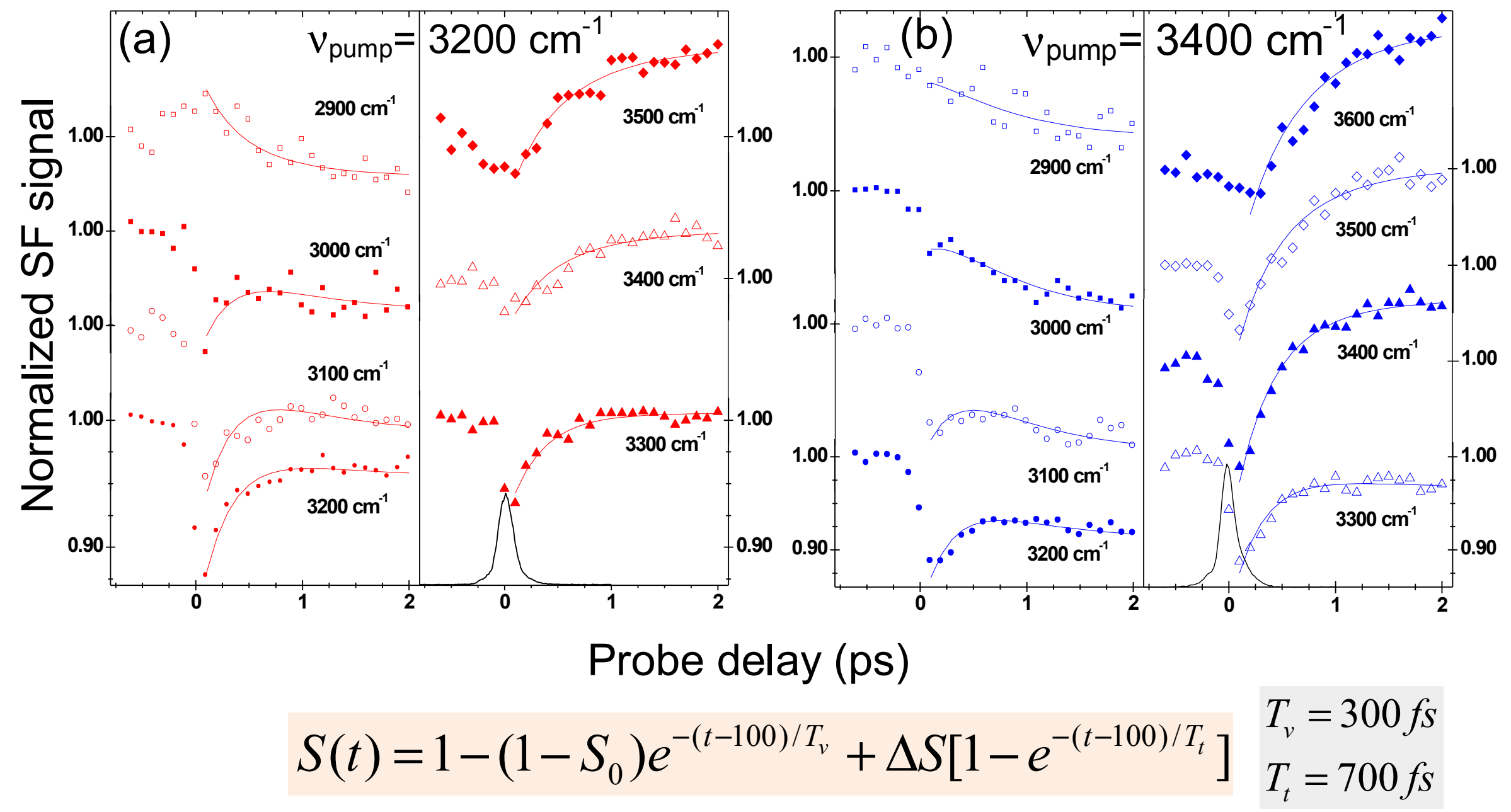




\section{OH Vibrational Relaxation}

- Excitations of $\mathrm{H}$-bonded $\mathrm{OH}$ stretches

- $t \sim 100 f s$ : Spectral diffusion of excitations to available $\mathrm{OH}$ stretches in the H-bonding network is over.

- $t \sim 300 f_{s}$ : Vibrational relaxation from $v=1$ states.

- $t \sim 700 f s$ : Thermalization of deposited energy. Temperature increase red-shifts the OH vibrational spectrum.

$$
\begin{gathered}
S(t)=1-\left(1-S_{0}\right) e^{-(t-100) / T_{v}}+\Delta S\left[1-e^{-(t-100) / T_{t}}\right] \\
\text { with } T_{v}=300 f_{S} \text { and } T_{t}=700 f_{S}
\end{gathered}
$$




\section{Advantages of SF Spectroscopy}

- Submonolayer sensitivity

- Highly -surface specific

- Output highly directional

- Non-detrimental, in situ, remote sensing

- High spatial, temporal, spectral resolution Surface microscopy, surface dynamics

- Applicable to any interfaces accessible by light many unique applications 


\section{Unique Applications}

- Buried interfaces

- Surface structures of neat bulk materials: polymers, liquids, etc.

- Molecular adsorption under ambient condition

- Surface dynamics, surface reactions

- Surface microscopy 


\section{Experimental Challenges}

- Extension to longer IR wavelengths.

- Better surface sensitivity

- Higher spatial resolution

- Probing surfaces of nanostructures

- Measuring depth profile of surface layer

- Accessing inaccessible interfaces 


\section{Theoretical Challenges}

- Assignment of spectral features

- Relating spectrum to interfacial structure
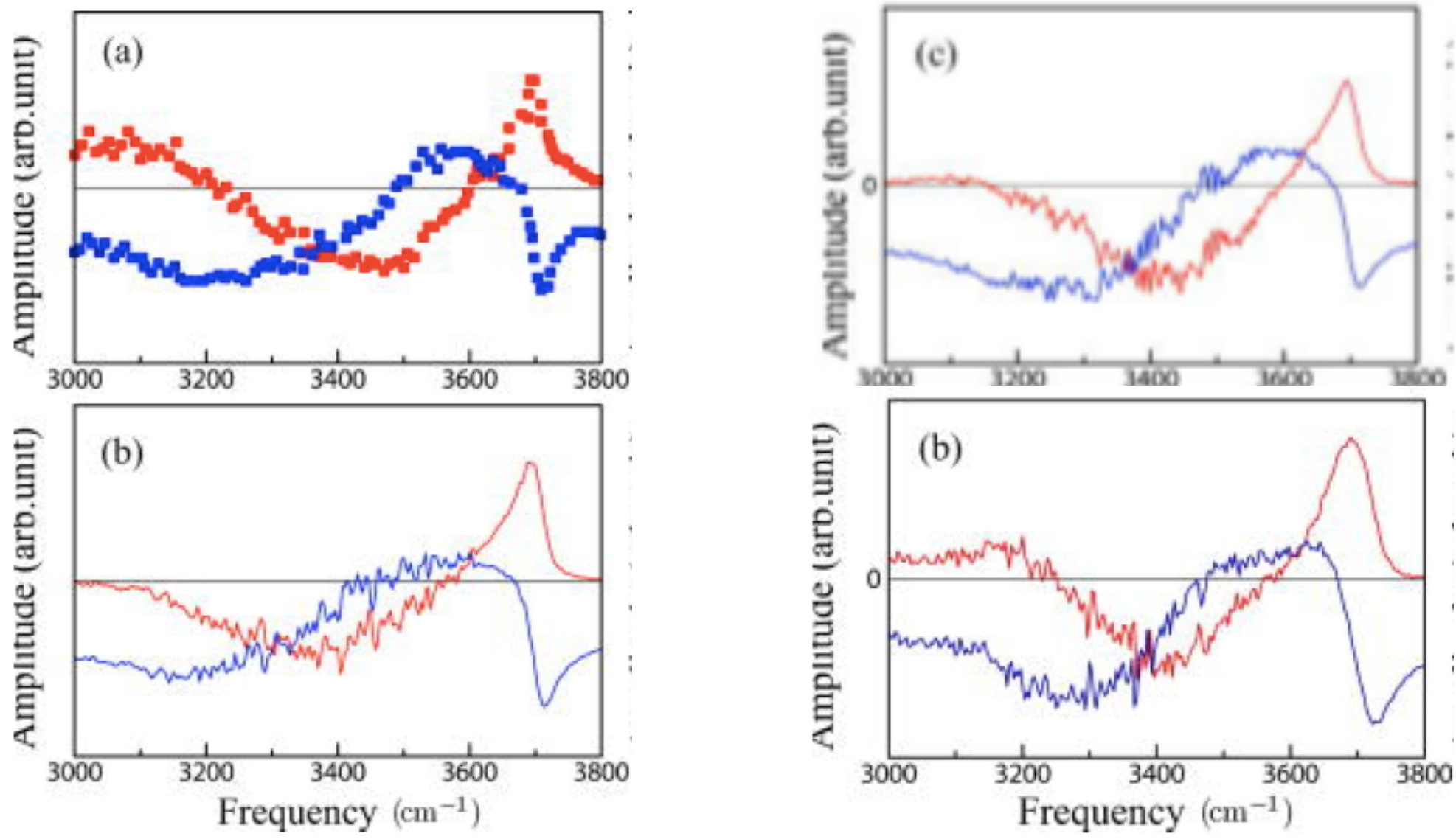


\section{Prospects}

- Studies of biological interfaces

- Understanding surface reactions at molecular level

Reactions at liquid/solid interfaces

Water splitting

Fuel cells

Photovoltaic cells

- Ultrafast surface dynamics

Energy transfer of surface excitations

Energy relaxation pathways

Control of surface reactions 
Acknowledgement

To All Who Have Contributed 